\title{
Modelling of optical emissions enhanced by the HF pumping of the ionospheric F-region
}

\author{
T. Sergienko ${ }^{1}$, B. Gustavsson ${ }^{2}$, U. Brändström ${ }^{1}$, and K. Axelsson ${ }^{1}$ \\ ${ }^{1}$ Swedish Institute of Space Physics, Kiruna, Sweden \\ ${ }^{2}$ Department of Physics and Astronomy, University of Southampton, UK \\ Correspondence to: T. Sergienko (tima@irf.se)
}

Received: 28 December 2011 - Revised: 4 May 2012 - Accepted: 7 May 2012 - Published: 31 May 2012

\begin{abstract}
Strong enhancement of the optical emissions with excitation threshold from $1.96 \mathrm{eV}\left(630.0 \mathrm{~nm}\right.$ from $\left.\mathrm{O}\left({ }^{1} \mathrm{D}\right)\right)$ up to $18.75 \mathrm{eV}\left(427.8 \mathrm{~nm}\right.$ from $\left.\mathrm{N}_{2}^{+}(1 \mathrm{NG})\right)$ have been observed during experiments of the ionosphere modification by high power HF radio waves. Analysis of the optical emission ratios showed clearly that a significant part of the ionospheric electrons have to be accelerated to energies above $30 \mathrm{eV}$ and more in the region where the HF radio wave effectively interacts with the ionospheric plasma. The Monte-Carlo model of electron transport and the optical emission model were used to study the dependence of the optical emission intensity on the acceleration electron parameters. We obtained the following results from analysis of the enhanced intensities of the four optical emissions (630.0, 557.7, 844.6 and $427.8 \mathrm{~nm}$ ) observed in the EISCAT heating experiment on 10 March 2002. The 630.0 emission with an excitation threshold of $1.96 \mathrm{eV}$ is formed predominately by the thermal electrons, where the accelerated electrons play a minor role in the excitation of this emission. In order to explain the experimentally observed intensity ratios, the accelerated electrons must gain energies of more than $60 \mathrm{eV}$. For accelerated electrons with a power law energy dependence, the efficiency of the optical emission excitation depends on the exponent defining the shape of the electron spectra. However, an agreement with the observed emission intensities is achieved for exponent values not less than zero. Moreover, increasing the exponent to higher values does not affect the emission intensity ratios.
\end{abstract}

Keywords. Ionosphere (Active experiments; Ionospheric disturbances)

\section{Introduction}

Strong enhancement of the optical emissions with excitation thresholds from $1.96 \mathrm{eV}\left(630.0 \mathrm{~nm}\right.$ from $\left.\mathrm{O}\left({ }^{1} \mathrm{D}\right)\right)$ up to $18.75 \mathrm{eV}\left(427.8 \mathrm{~nm}\right.$ from $\left.\mathrm{N}_{2}^{+}(1 \mathrm{NG})\right)$ have been observed during experiments of ionosphere modification by high power HF radio waves since the early 1970s (Biondi et al., 1970; Sipler et al., 1974; Adeishvili et al., 1979; Bernhardt et al., 1988; Brändström et al., 1999; Pedersen and Carlson, 2001; Kosch et al., 2005; Djuth et al., 2005; Gustavsson et al., 2005; Holma et al., 2006). It has also been well known that powerful $\mathrm{HF}$ radio wave transmission leads to strong enhancement of the temperature of the ionospheric electrons. Mantas and Carlson (1996) developed a quantitative model relating to the heating enhanced electron temperature to the $630.0 \mathrm{~nm}$ intensity and argued that the thermal electron excitation of the $\mathrm{O}\left({ }^{1} \mathrm{D}\right)$ state is enough to explain the emission intensity observed during the ionosphere heating experiments. However, in an analysis of simultaneous observations of the electron density, temperature and the red line intensity, Sergienko et al. (2000) showed that the model significantly overestimates the observed emission intensity. In order to explain this discrepancy, the authors proposed that inelastic collisions with atmospheric gases modify the shape of the electron distribution function. This idea was proved in papers by Mishin et al. (2000) and Gustavsson et al. (2004). In those studies it was shown that for F-region night-time conditions, excitation of the vibrational states of $\mathrm{N}_{2}$ causes a strong depletion in the electron distribution in the high $(E>2 \mathrm{eV})$ energy range resulting in a decrease in the production rate of the $\mathrm{O}\left({ }^{1} \mathrm{D}\right)$ state.

Although such modification of the thermal electron distribution function partly explains the discrepancies between modelled and observed intensities of the $630.0 \mathrm{~nm}$ emission, 
the observed intensities of the emissions with higher thresholds cannot be explained solely via excitation by the thermal electrons. For instance, the observation of the heating induced emission at $427.8 \mathrm{~nm}$ with an excitation threshold of $18.75 \mathrm{eV}$ indicates that electrons are accelerated to energies higher than $20 \mathrm{eV}$. The most probable mechanism for such acceleration is resonant interaction with the plasma waves stimulated by the nonlinear interaction of the powerful HF radio wave with the ionospheric plasma. It is still an open question which wave-plasma instabilities are driving the electron energization. It has been debated (see Grach, 1999; Mishin et al., 2005; Gurevich, 2007, and references therein) whether Langmuir turbulence or the upper hybrid plasma turbulence is the most prominent electron acceleration mechanism. As shown by Gustavsson and Eliasson (2008), analysis of the heating induced optical emissions originating from the excited states with different thresholds and with significantly different excitation cross-sections provides a potential opportunity for estimation of the shape of the differential flux of the accelerated electrons. Such information will be useful for the identification of the acceleration mechanism.

In this paper, we have applied the Monte Carlo method for the simulation of the transport of the supra thermal electrons into the upper Earth atmosphere. The calculated electron differential fluxes were used for analysis of the excitation mechanisms of the optical emission enhancement stimulated by the high power radio wave in the ionospheric F-region. Two simplified models for the acceleration electron source were assumed in order to investigate the dependence of the optical emission intensity on the acceleration electron parameters.

\section{Model description}

\subsection{Model of optical emissions}

This study compares modelling results with experimental data obtained during an EISCAT heating experiment conducted on 10 March 2002. The ionospheric parameters (electron density, and electron and ion temperatures) were measured by the UHF EISCAT radar simultaneously with the optical observations by ALIS of enhancements in the 427.8, 557.7, 630.0 and $844.6 \mathrm{~nm}$ emissions. A detailed description of this experiment and observations have been provided by Gustavsson et al. (2005). A comprehensive analysis of the heating induced enhancement of the optical emissions was done by Gustavsson and Eliasson (2008). The optical emission model developed in that paper is adopted for the present study with a few minor revisions which will be given in this section.

${ }^{1} \mathrm{D}$ state of atomic oxygen has a long radiative lifetime ( $\sim 130 \mathrm{~s}$ ) and is quenched in collisions with the neutral atmospheric particles as well as with the thermal electrons. In this study the quenching rate of $\mathrm{O}\left({ }^{1} \mathrm{D}\right)$ state is calculated by taking into account the following reactions with $\mathrm{N}_{2}, \mathrm{O}_{2}, \mathrm{O}$ and with thermal electrons (Berrington and Burke, 1981):

$\mathrm{O}\left({ }^{1} \mathrm{D}\right)+\mathrm{N}_{2} \stackrel{k_{1}}{\rightarrow} \mathrm{O}\left({ }^{3} \mathrm{P}\right)+\mathrm{N}_{2}$

$\mathrm{O}\left({ }^{1} \mathrm{D}\right)+\mathrm{O}_{2} \stackrel{k_{2}}{\rightarrow} \mathrm{O}\left({ }^{3} \mathrm{P}\right)+\mathrm{O}_{2}$

$\mathrm{O}\left({ }^{1} \mathrm{D}\right)+\mathrm{O} \stackrel{k_{3}}{\rightarrow} \mathrm{O}\left({ }^{3} \mathrm{P}\right)+\mathrm{O}\left({ }^{3} \mathrm{P}\right)$

$\mathrm{O}\left({ }^{1} \mathrm{D}\right)+e_{\text {th }} \stackrel{k_{4}}{\rightarrow} \mathrm{O}\left({ }^{3} \mathrm{P}\right)+e_{\text {th }}$

with respective quenching rate coefficients,

$k_{1}=2.0 \times 10^{-11} \exp \left(\frac{107.8}{T_{\mathrm{n}}}\right), \mathrm{cm}^{3} \mathrm{~s}^{-1}$,

(Streit et al., 1974)

$k_{2}=2.9 \times 10^{-11} \exp \left(\frac{67.5}{T_{\mathrm{n}}}\right), \mathrm{cm}^{3} \mathrm{~s}^{-1}$,

(Streit et al., 1974)

$k_{3}=2.2 \times 10^{-11}, \mathrm{~cm}^{3} \mathrm{~s}-1$,

(Kalogerakis et al., 2009)

$k_{4}=1.6 \times 10^{-12} T_{\mathrm{e}}^{0.91}, \mathrm{~cm}^{3} \mathrm{~s}^{-1}$,

(Berrington and Burke, 1981). Here $T_{\mathrm{n}}$ and $T_{\mathrm{e}}$ are neutral and electron temperature, respectively. Finally the $630.0 \mathrm{~nm}$ volume emission rate is calculated by the formula:

$V_{6300}=\frac{Q_{{ }_{1} \mathrm{D}} \cdot A_{6300}}{A_{{ }_{1} \mathrm{D}}+k_{1}\left[\mathrm{~N}_{2}\right]+k_{2}\left[\mathrm{O}_{2}\right]+k_{3}[\mathrm{O}]+k_{4}\left[N_{\mathrm{e}}\right]}$

Here $A_{6300}$ and $A_{1_{D}}$ are the Einstein coefficients for the $630.0 \mathrm{~nm}$ emission and the sum of the Einstein coefficients for all emissions from $\mathrm{O}\left({ }^{1} \mathrm{D}\right)$ state. Values for these coefficients, as well as for other Einstein coefficients which are used in this model, are given in Table $1 ;[M]$ is the number density of corresponding atmospheric species or thermal electrons. Here, as well as in the following equations, $Q_{i}$ represents the production rate of the excited state due to electron impact.

For the $844.6 \mathrm{~nm}$ emission, Gustavsson and Eliasson (2008) took into account the direct electron excitation of $\mathrm{O}\left(3 p^{3} P\right)$ state only. Here we also include the excitation of this state due to radiative transfer from the upper excited states of $\mathrm{O}$ since this mechanism gives about $30 \%$ of the total $\mathrm{O}\left(3 p^{3} P\right)$ production. Thus, the $844.6 \mathrm{~nm}$ volume emission rate is calculated using the equation:

$$
\begin{aligned}
V_{8446}= & \left(Q_{3 p^{3} P}+Q_{3 s^{\prime 3} D^{0}} \frac{A_{7992}}{A_{3 s^{\prime 3} D^{0}}}\right. \\
& \left.+Q_{3 d^{3} D^{0}} \frac{A_{11278}}{A_{3 d^{3} D^{0}}}+Q_{3 s^{\prime \prime 3} P^{0}} \frac{A_{3953}}{A_{3 s^{\prime \prime 3} P^{0}}}\right)
\end{aligned}
$$

Here $A_{7990}$ and $A_{11278}$ and $A_{3953}$ are the Einstein coefficients for corresponding emissions and $A_{3 p^{3} P}, A_{3 s^{\prime 3} D^{0}}$ and 
Table 1. Einstein coefficients.

\begin{tabular}{ccl}
\hline & Einstein coefficient, s & Reference \\
\hline$A_{6300}$ & $5.627 \times 10^{-3}$ & Itikawa and Ichimura (2090) \\
$A_{1} \mathrm{D}$ & $7.446 \times 10^{-3}$ & Itikawa and Ichimura (2090) \\
$A_{5577}$ & 1.215 & Itikawa and Ichimura (2090) \\
$A_{1} \mathrm{~S}$ & 1.291 & Itikawa and Ichimura (2090) \\
$A_{7992}$ & $0.562 \times 10^{5}$ & Ralchenko et al. (2011) \\
$A_{3 s^{\prime 3} D^{0}}$ & $0.115 \times 10^{6}$ & Ralchenko et al. (2011) \\
$A_{11278}$ & $0.309 \times 10^{8}$ & Ralchenko et al. (2011) \\
$A_{3 d^{3} D^{0}}$ & $0.107 \times 10^{9}$ & Ralchenko et al. (2011) \\
$A_{3953}$ & $0.309 \times 10^{6}$ & Ralchenko et al. (2011) \\
$A_{3 s^{\prime \prime 3} P^{0}}$ & $0.341 \times 10^{6}$ & Ralchenko et al. (2011) \\
$A_{4278}$ & $0.371 \times 10^{7}$ & Gilmore et al. (1992) \\
$A_{B_{v=0}^{2} \Sigma_{u}^{+}}$ & $0.161 \times 10^{8}$ & Gilmore et al. (1992) \\
\hline
\end{tabular}

$A_{3 d^{3} D^{0}}$ and $A_{3 s^{\prime \prime 3} P^{0}}$ are the sums of the Einstein coefficients for all emissions from corresponding states of atomic oxygen. For the 427.8 and $557.7 \mathrm{~nm}$ emissions, their volume emission rates are given by the equations:

$V_{4278}=Q_{B^{2} \Sigma_{u}^{+}} \cdot q_{0 \rightarrow 0} \frac{A_{4278}}{A_{B^{2} \Sigma_{u(v=0)}^{+}}}$

$V_{5577}=Q_{{ }_{1} \mathrm{~S}} \frac{A_{5577}}{A_{{ }_{1} \mathrm{~S}}}$

Here $q_{(0 \rightarrow 0)}=0.883$ is the Franck-Condon factor for the electronic-vibrational transitions from vibrational level $v=0$ of the ground state of $\mathrm{N}_{2}\left(X^{1} \Sigma_{g}^{+}\right)$to the vibrational level $v=0$ of $B^{2} \Sigma_{u}^{+}$state of $\mathrm{N}_{2}{ }^{+}$(Gilmore et al., 1992), and $A_{4278}, A_{B^{2} \Sigma_{u(v=0)}^{+}}, A_{5577}$ and $A_{1 \mathrm{~S}}$ are the corresponding Einstein coefficients of molecular nitrogen ion and atomic oxygen, respectively. The production rate $Q$ in Eqs. (1)-(4) is given by the integral

$Q(z)=[M(z)] \cdot \int_{E_{\mathrm{th}}}^{\infty} \sigma(E) F(E, z) d E$

where $z$ is altitude, $(E)$ is electron cross-section for the excitation of corresponding electronic level of $\mathrm{N}_{2}$ and $\mathrm{O}, F(E, z)$ is the omnidirectional differential number flux of the super thermal electrons at altitude $z$, and $E_{\text {th }}$ is the excitation threshold. Excitation cross-sections used in the optical emission model are presented in Fig. 1. The differential flux $F(E, z)$ is calculated with the Monte Carlo model of electron transport into the upper atmosphere, described in the following section. Finally, the column intensities of the emissions can be calculated by integration of the volume emission rates over the altitude $z$

$I=10^{-6} \int V(z) d z$

where $I$ is the intensity in Rayleighs, $V(z)$ is the volume emission rate in $\left(\mathrm{cm}^{-3} \mathrm{~s}^{-1}\right)$ and $z$ is the altitude in centimetres.

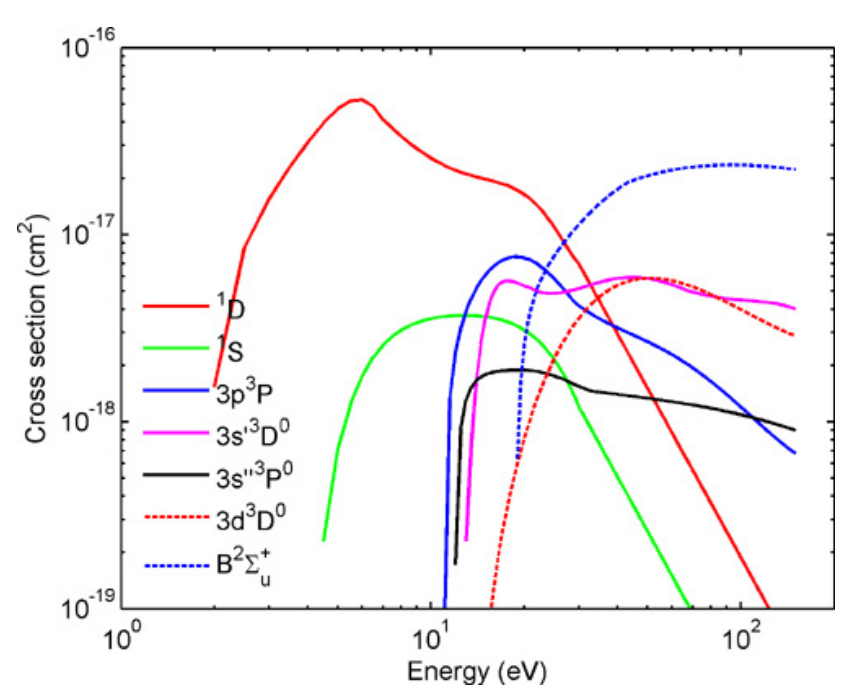

Fig. 1. Excitation cross-sections of optical emissions used in the model.

\subsection{The electron transport model}

For modelling of the transport of the suprathermal $(E>$ $1 \mathrm{eV}$ ) ionospheric electrons, we adopted the Monte Carlo model developed for auroral electron transport described in detail by Sergienko and Ivanov (1991). In this model, the electron trajectory is treated as a sequence of elastic and inelastic collisions with the atmospheric atoms and molecules, between which the electron moves under the influence of the homogeneous magnetic field as well as the drag force due to interaction with the ambient (cold) thermal electrons. The distance that an electron will move between two successive collisions, the collision type, the new electron velocity direction after the collision, and an energy of the secondary electron are determined randomly according to the corresponding probabilities defined by the scattering cross-sections and the number density of the atmospheric gases $\left(\mathrm{N}_{2}, \mathrm{O}_{2}\right.$, and $\left.\mathrm{O}\right)$. For the neutral densities we use the MSIS atmosphere model (Hedin, 1991). Integral cross-sections of elastic and inelastic electron collisions with the atomic oxygen are taken from the review by Laher and Gilmore (1990). Cross-sections of electron-molecular nitrogen collisions used in our model are similar to cross-sections presented by Tabata et al. (2006). Electron cross-sections for molecular oxygen are taken from the review by Itikawa (2009).

To calculate a new velocity direction of an electron, after an elastic collision, we use the differential elastic crosssections expressed in parametric form by Porter et al. (1987). Energy of a secondary electron resulting from the ionizing collision is calculated using the analytical representation (Jackman et al., 1977) of the double differential ionization cross-section measured in a laboratory experiment by Opal et al. (1971). Continuous energy loss of the streaming electrons, due to the interaction with the cold electron gas, is 
Table 2. Ionospheric parameters and optical emission intensities measured during the EISCAT heating experiment on 2 March 2002.

\begin{tabular}{lccccccccc}
\hline & $\begin{array}{c}\max \left(N_{\mathrm{e}}\right), \\
\mathrm{cm}^{-3}\end{array}$ & $\begin{array}{c}\max \left(T_{\mathrm{e}}\right), \\
\mathrm{K}\end{array}$ & $\begin{array}{c}\mathrm{h}\left(\max \left(N_{\mathrm{e}}\right)\right), \\
\mathrm{km}\end{array}$ & $\begin{array}{c}\mathrm{h}\left(\max \left(T_{e}\right)\right), \\
\mathrm{km}\end{array}$ & $\begin{array}{c}\mathrm{h}\left(f_{\mathrm{HF}}\right), \\
\mathrm{km}\end{array}$ & $\begin{array}{c}I_{6300}, \\
\mathrm{R}\end{array}$ & $\begin{array}{c}I_{5577}, \\
\mathrm{R}\end{array}$ & $\begin{array}{c}I_{8446}, \\
\mathrm{R}\end{array}$ & $\begin{array}{c}I_{4278}, \\
\mathrm{R}\end{array}$ \\
\hline Pulse 1 18:12 UT & $6.6 \times 10^{6}$ & 3330 & 320 & 227 & 230 & 240 & 43 & 35 & 7 \\
Pulse 2 18:54 UT & $5.2 \times 10^{6}$ & 3550 & 360 & 260 & 270 & 420 & 47 & 53 & 7 \\
\hline
\end{tabular}
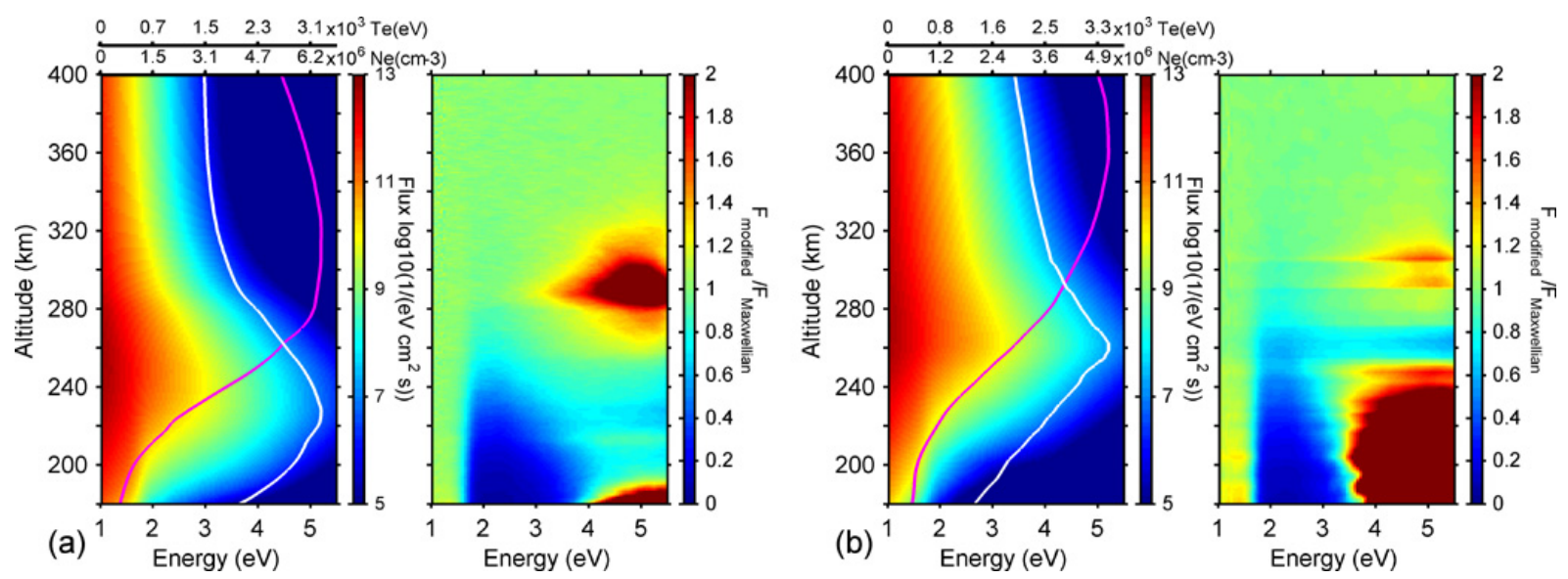

Fig. 2. (a) Left: Altitude profile of the differential flux of the high energy tail of the ionospheric thermal electrons for ionospheric conditions corresponding to the heating pulse at 18:12 UT on 10 March 2002. The colour lines, white and magenta, present the altitude profiles of the ionospheric electron temperature and density, respectively. Right: Altitude energy variation of the ratio of the modelled differential electron flux to the Maxwellian flux; (b) same as panel (a) for the heating pulse at 18:54 UT.

determined using the loss function derived by Swartz et al. (1971).

An electron trajectory (primary and secondary) is traced until the electron exits the upper boundary of the atmosphere or reaches energy of less than $1 \mathrm{eV}$. The model operates in the altitude range of 150-700 km with an altitude step of $0.2 \mathrm{~km}$. A differential electron flux is tabulated by counting each altitude level crossing within each energy and pitch angle bin, and is normalized to the total particle flux according to a particular form of the initial electron source. The specific form of the source for the high energy tail of the thermal electrons and for the accelerated electrons will be given in the related sections.

\section{Results}

On 10 March 2002 the EISCAT Heating Facility was operated in a $4 \mathrm{~min}$ on and $2 \mathrm{~min}$ off cycle at $5.423 \mathrm{MHz}$ transmitting $290 \mathrm{MW}$ effective radiated power in ordinary mode to enhance airglow in the nighttime F-region ionosphere. The enhanced optical emissions were observed during 15 consecutive heating pulses (see Fig. 2 in Gustavsson et al., 2005). These pulses can be divided into two groups according to the intensity of $630.0 \mathrm{~nm}$ emission, five pulses at the beginning of the experiment and eight pulses at the end. The enhanced emission intensity within each group was approxi- mately constant, but it increased almost by a factor of two for $630.0 \mathrm{~nm}$ emission in the transition from the first group of pulses to the second group. This intensity increase occurred at the same time as the electron density suddenly dropped and, consequently, the reflection height of the HF pumping radio wave increased from $230 \mathrm{~km}$ to $270 \mathrm{~km}$. For further analysis, we selected one pulse from each group. The selection criterion was the smallest variations of the ionospheric parameters and of the emission intensities during the pulse. Table 2 summarizes important values observed during the selected pulses: the maximum value of electron density and temperature, the altitude of these maxima, the altitude of the HF radio wave reflection, and the intensities of 630.0, 557.7, 844.6 and $427.8 \mathrm{~nm}$ emissions. We must note that the values of the optical emission intensities listed in Table 2 differ from the values given in Fig. 5 in the paper by Gustavsson et al. (2005). These differences are due to an error in the algorithm used to plot Figs. 2, 3 and 5 in Gustavsson et al. (2005). The relative errors of the emission intensities due to uncertainty in the absolute calibration of ALIS camera and a scattering of the experimental data are $25 \%$ for the $630.0,557.7$ and $844.6 \mathrm{~nm}$ emissions and $35 \%$ for the $427.8 \mathrm{~nm}$ emission. The altitude profiles of the electron density and temperature are shown in Fig. 2a and b for Pulse 1 and Pulse 2, respectively. 

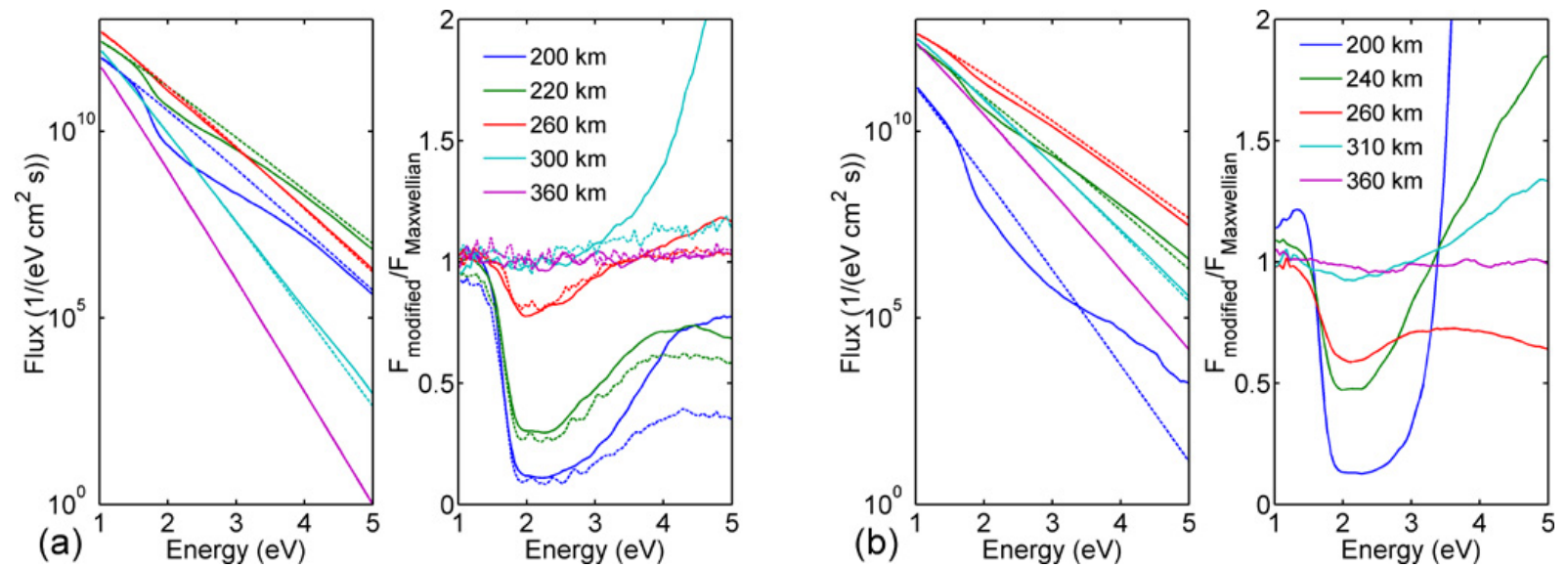

Fig. 3. (a) The differential flux (left) and ratio of the differential fluxes (right) at different altitudes for the heating pulse at 18:12 UT. The dashed lines on the left plot show the corresponding Maxwellian fluxes. The dashed lines on the right plot show calculation results obtained without electron transport effects; (b) same as panel (a) for the heating pulse at 18:54 UT.
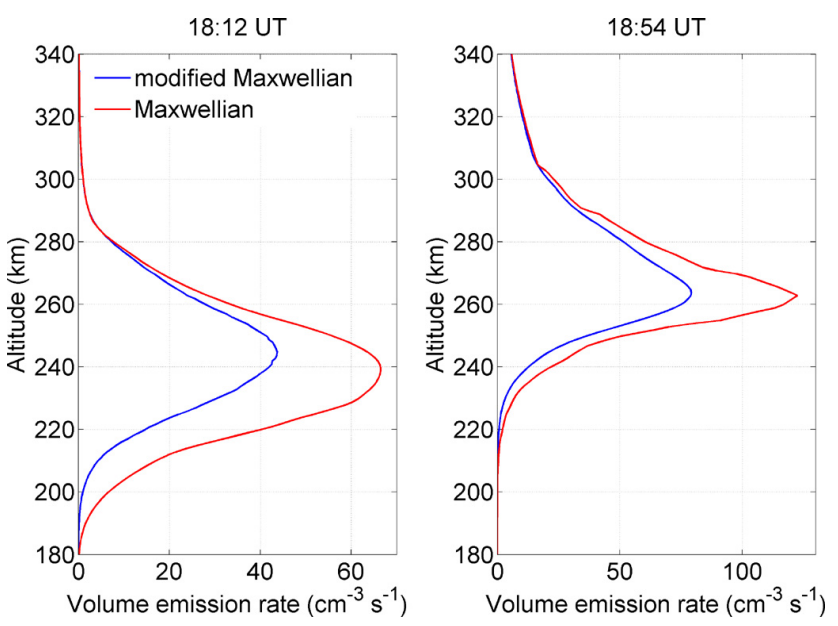

Fig. 4. The altitude profile of the $630.0 \mathrm{~nm}$ volume emission rate. The blue line corresponds to the electron flux calculated with the transport model. The red line shows the calculated result with a Maxwellian flux of the ionospheric electrons.

\subsection{High energy tail of the thermal electrons}

The $\mathrm{O}\left({ }^{1} \mathrm{D}\right)$ state emitting at $630.0 \mathrm{~nm}$ has an excitation threshold of $1.96 \mathrm{eV}$ and it can be effectively excited by the high energy tail of the ionospheric thermal electrons. Mantas and Carlson (1996) developed a quantitative model relating to the heating enhanced electron temperature to the $630.0 \mathrm{~nm}$ intensity and showed that the thermal electron excitation of the $\mathrm{O}\left({ }^{1} \mathrm{D}\right)$ state is enough to explain the emission intensity observed during the ionosphere heating experiments.

In the model, Mantas and Carlson (1996) assumed that the thermal electrons have a pure Maxwellian distribution function. However, in an analysis of simultaneous observations of the electron density, temperature and the red line inten- sity, Sergienko et al. (2000) showed that this model significantly overestimates the observed emission intensity. In order to explain this discrepancy, the authors proposed that the inelastic collisions with atmospheric gases modify the shape of the electron distribution. This idea was proved in papers by Mishin et al. (2000) and Gustavsson et al. (2004). In those studies, it was shown that for F-region night-time conditions excitation of the vibrational states of $\mathrm{N}_{2}$ causes a strong depletion in the electron distribution in the high $(E>2 \mathrm{eV})$ energy range that resulted a decrease of the production rate of the $\mathrm{O}\left({ }^{1} \mathrm{D}\right)$ state. Both of the above mentioned models run in the local-loss approximation and transport effects were ignored.

This section presents the results of the modelling study of (1) an effect of the electron transport on the collisional modification of the high energy tail of the thermal electron distribution, and (2) a contribution of the thermal electrons to total enhancement of the $630.0 \mathrm{~nm}$ intensity. In order to run our transport model, it is necessary to determine the source of the electrons. In the steady state and neglecting the external sources that we consider here, the only source of the high energy tail of the thermal electrons is the "thermalization" of the electron gas. For linear approximation in the equilibrium state, the kinetic equation for the high energy thermal electrons can be written as:

$N_{\mathrm{e}} \frac{\partial}{\partial E}(L(E) \cdot F(E))=P(E)$

where $P(E)$ is the source function of the high energy electrons, $L(E)$ is the loss function representing a continuous energy loss due to interaction of the streaming electrons with the cold electron gas as given by Swartz et al. (1971):

$L(E)=\frac{3.37 \times 10^{-12}}{E^{0.94} N_{\mathrm{e}}^{0.03}}\left(\frac{\left(E-T_{\mathrm{e}}\right)}{\left(E-0.53 T_{\mathrm{e}}\right)}\right)^{2.36}, \mathrm{~cm}^{2} \mathrm{eV}$ 

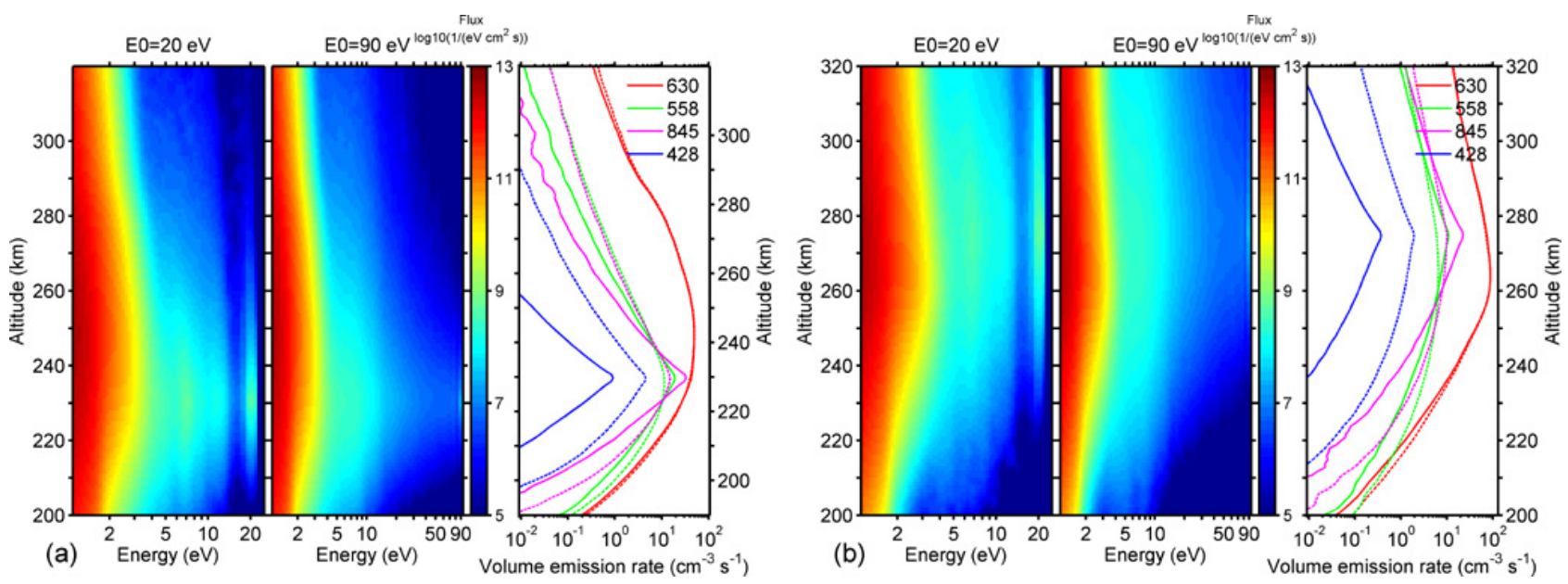

Fig. 5. (a) The electron differential flux and the volume emission rate for the heating pulse at 18:12 UT. Left: The altitude energy variation of the electron flux for the mono-energetic accelerated electrons with an initial energy of $20 \mathrm{eV}$. Centre: The same, but for an initial energy of $90 \mathrm{eV}$. Right: Profiles of volume emission rates of the $630.0 \mathrm{~nm}$ (red), $557.7 \mathrm{~nm}$ (green), $844.6 \mathrm{~nm}$ (magenta) and $427.8 \mathrm{~nm}$ (blue) optical emissions. The solid lines correspond to an accelerated electron energy of $20 \mathrm{eV}$, the dashed lines correspond to an energy of $90 \mathrm{eV}$; (b) same as Fig. 7 for the heating pulse at 18:54 UT.
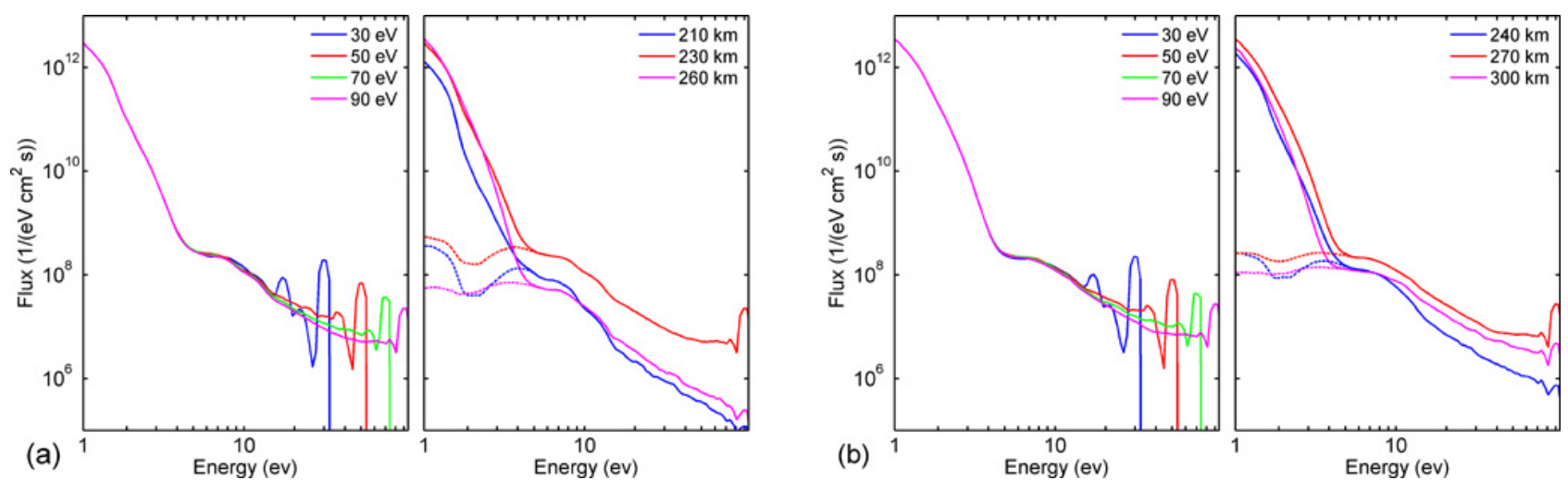

Fig. 6. (a) The energy dependence of the electron differential flux for the heating pulse at 18:12 UT. Left: The differential fluxes for various energies of the accelerated electrons at the electron acceleration altitude $(230 \mathrm{~km})$. Right: The electron differential fluxes at various altitudes calculated for the acceleration energy of $90 \mathrm{eV}$. The dashed lines show the differential flux formed solely by the accelerated electrons; (b) same as panel (a) for the heating pulse at 18:54 UT.

Here $E$ is the electron energy in $\mathrm{eV}, N_{\mathrm{e}}$ is the electron density in $\mathrm{cm}^{-3}$ and $T_{\mathrm{e}}$ is the ambient electron temperature in eV. $F(E)$ in Eq. (5) is the electron flux with the Maxwellian distribution function:

$F(E)=\frac{2 N_{\mathrm{e}}}{\sqrt{\pi}\left(k T_{\mathrm{e}}\right)^{\frac{3}{2}}} \sqrt{\frac{2 e}{m_{\mathrm{e}}}} \times E \exp \left(-\frac{E}{k T_{\mathrm{e}}}\right), \mathrm{eV}^{-1} \mathrm{~cm}^{-2} \mathrm{~s}^{-1}$

Here $k$ is Boltzmann's constant and $m_{\mathrm{e}}$ is electron mass. Evaluation of Eq. (5) in each altitude bin gives the probability function for determination of the pseudo-random initial energy of electrons. The initial altitude of the electron is uniformly distributed within each altitude bin, and the pitch angle is assumed to be isotropically distributed. 5000 initial electrons in the energy range of $1-6 \mathrm{eV}$ in each altitude bin are used for a particular run to obtain reasonable statistics in the modelling electron spectrum.

The modelling results for the conditions of Pulse 1 and Pulse 2 are presented in Figs. 2a and 3a and Figs. 2b and 3b, respectively. The left panels of Fig. $2 a$ and $b$ show the calculated differential fluxes of the thermal electrons as a function of electron energy and altitude. The colour lines, magenta and white, present experimental electron density and temperature used for the modelling. The right panels of these figures present the energy-altitude distribution of the ratio of the electron differential flux obtained from our simulation to the Maxwellian flux defined by the formula (6). Figure 3a and $b$ presents the calculated and Maxwellian fluxes and their ratio at five different altitudes. 

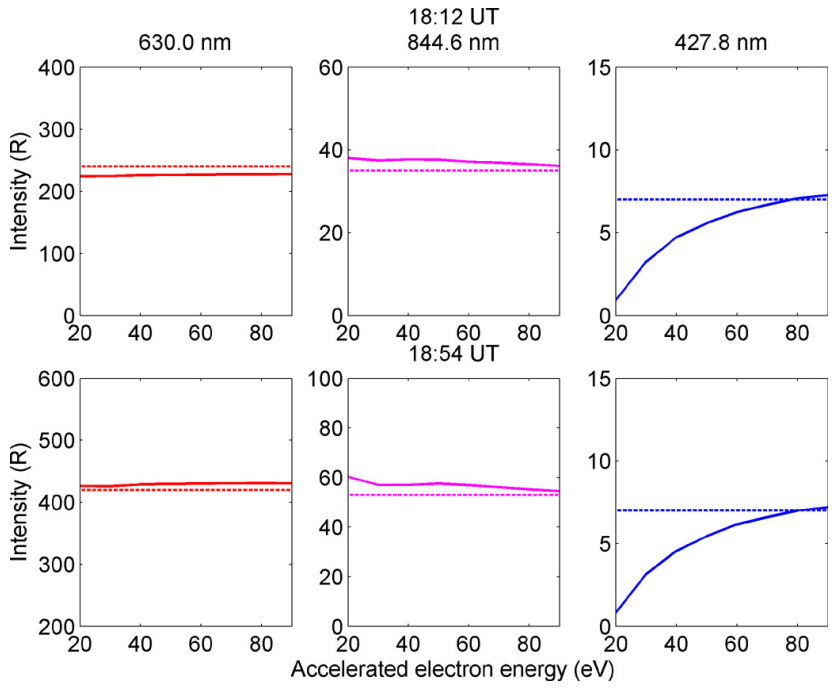

Fig. 7. The emission intensity dependence on energy of the accelerated electrons. The top row corresponds to the heating pulse at 18:12 UT. The bottom row corresponds to 18:54 UT. The solid lines show the calculated emission intensities. The dashed lines indicate the values of the emission intensities observed in the experiment. $I_{6300}^{\bmod } \equiv I_{6300}^{\mathrm{exp}}$.

As expected the differential electron flux at energy $E>$ $2 \mathrm{eV}$ decreases significantly due to excitation of the vibrational levels of $\mathrm{N}_{2}$. Our new result is clearly seen in the right panels of Fig. 2a and b, a dramatic enhancement of the electron flux at energies higher than $3.5 \mathrm{eV}$, at altitudes of a few tens of kilometres above and below the height of the maximum temperature. Since such a flux increase was not observed in the studies by Mishin et al. (2000) and Gustavsson et al. (2004), we attribute it as an effect of the electron transport. In order to prove this suggestion, we modified the transport model to run it under a local loss approximation. The ratios of the fluxes calculated with the modified code to the Maxwellian flux are showed as the dashed lines on the right plot in Fig. 3a and verify the electron transport effect.

Another result of the particle transport is manifested as an altitude shift of the largest electron flux relative to the maximum of the electron temperature. This difference is more noticeable for Pulse 2 because the higher the altitude, the longer the electron free path.

The $630.0 \mathrm{~nm}$ volume emission rates calculated with the modelled and with Maxwellian fluxes are plotted in Fig. 4. The emissions from the modified fluxes are significantly weaker than emission from the Maxwellian flux. Moreover, the altitude of the peak of the first one is shifted up due to the effect of the electron transport. The intensities of the $630.0 \mathrm{~nm}$ emission from Maxwellian flux are equal to $317 \mathrm{R}$ for Pulse 1 and to $483 \mathrm{R}$ for Pulse 2. These values are considerably larger than those observed experimentally. On the other hand, the emission intensities calculated from the modelled electron flux are $192 \mathrm{R}$ and $366 \mathrm{R}$, that are smaller than the experimental values by $48 \mathrm{R}$ for Pulse 1 and by $54 \mathrm{R}$ for Pulse 2. In the next section, we will see whether it is possible to explain this difference by the presence of accelerated particles.

\subsection{Accelerated electrons}

In contrast to the $630.0 \mathrm{~nm}$ emission, the excitation by thermal electrons cannot explain the observation of enhancements in emissions from states with an excitation thresholds. The observation of the heating induced emission at $427.8 \mathrm{~nm}$ with the excitation threshold $18.75 \mathrm{eV}$ indicates that electrons are accelerated to energies higher than $20 \mathrm{eV}$ via resonance interaction with the plasma waves stimulated by the nonlinear interaction of the powerful HF radio wave with the ionospheric plasma. The Langmuir turbulence and the upper hybrid plasma turbulence are debated in literature as the most prominent candidates for the electron acceleration mechanisms (see Grach, 1999; Mishin et al., 2005; Gurevich, 2007, and references therein).

In the present study, in order to define a source of the accelerated electrons for Monte Carlo simulation, we adopt two simplified models. The first model assumes the existence of the field aligned electrostatic structure within a narrow altitude region at an altitude just below the reflection height of the pumping HF radio waves $\left(h\left(f_{\text {fpump }}\right)\right.$ in Table 2 . For such a model, the initial electron energy assigned for a particular run of the electron transport code is defined by the following source distribution function:

$P\left(E_{0}\right)=A \int_{E} \delta\left(E-E_{0}\right) d E, \mathrm{~cm}^{-3} \mathrm{~s}^{-1}$

Here $E_{0}$ is the initial energy of accelerated electrons which is equal to the value of the electrostatic potential in the centre of the acceleration region, $\delta\left(E-E_{0}\right)$ is the Dirac delta function and $A$ is a constant that will be defined later. In the first model, all electrons are accelerated to the same energy in both directions, up and down.

For the second model it is suggested that within the same altitude range there is electrostatic turbulence which results in the accelerated electron flux with a power-law dependence on energy. For this acceleration source the energy distribution function of the accelerated electrons is given by:

$P\left(E_{0}\right)=A \int_{1}^{E_{0}} E^{\gamma} d E, \mathrm{~cm}^{-3} \mathrm{~s}^{-1}$

In this model the initial energy, $E_{0}$, of the accelerated electrons varies in the energy range of $1-100 \mathrm{eV}$ and the pitch angle distribution of the electrons is suggested to be isotropic. Neither model is attributed to any real acceleration mechanisms, but they allow us to investigate the dependence of the optical emission enhancement on the accelerated electron energy and of the shape of their spectra. 

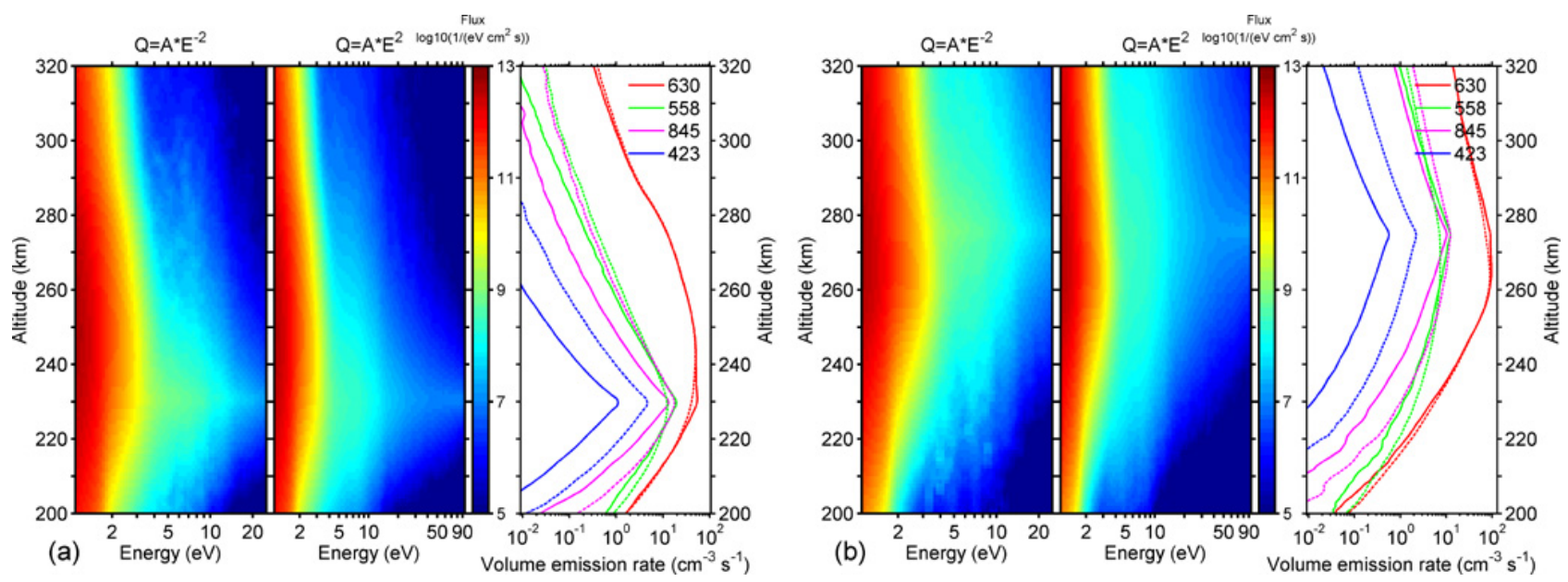

Fig. 8. (a) The electron differential flux and the volume emission rate for the heating pulse at 18:12 UT. Left: Altitude energy variation of the electron flux for the power-law acceleration electron source with the exponent -2 . Centre: Same as the left panel with the exponent 2 . Right: Volume emission rate profiles of the $630.0 \mathrm{~nm}$ (red), $557.7 \mathrm{~nm}$ (green), $844.6 \mathrm{~nm}$ (magenta) and $427.8 \mathrm{~nm}$ (blue) optical emissions. The solid lines correspond to the electron acceleration source with the exponent -2 , the dashed lines corresponds to the exponent 2; (b) same as panel (a) for the heating pulse at 18:54 UT.
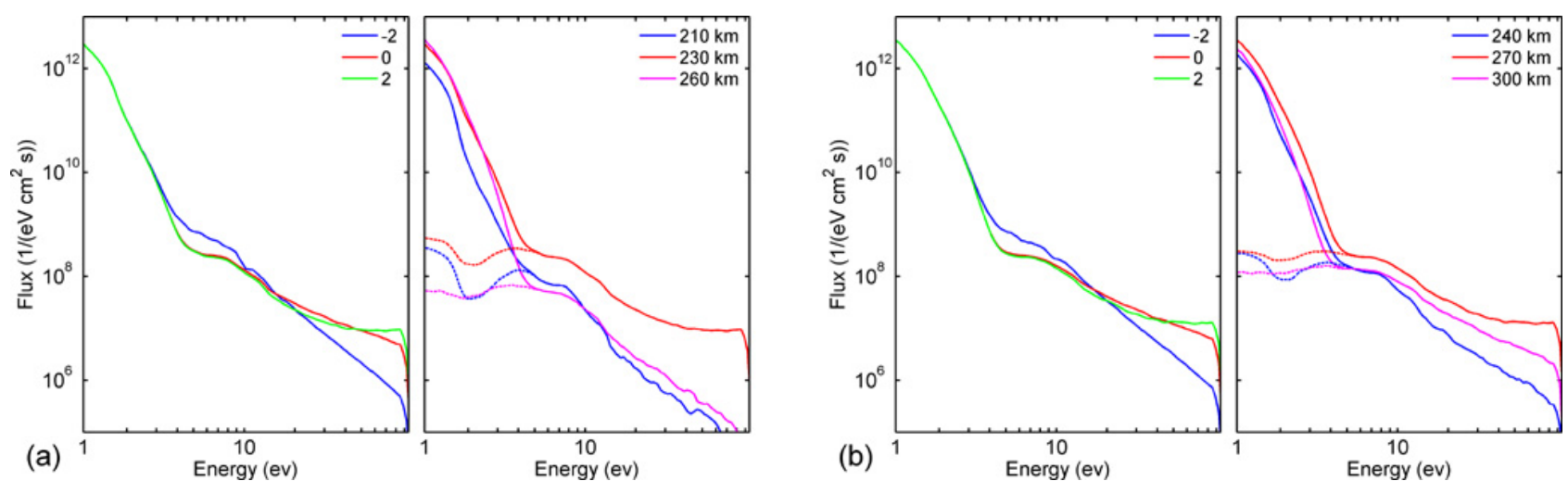

Fig. 9. (a) Energy dependence of the electron differential flux for the heating pulse at 18:12 UT. Left: The differential electron fluxes for the power-law acceleration source with the different exponents at the electron acceleration altitude ( $230 \mathrm{~km})$. Right: The electron differential fluxes at various altitudes calculated for the acceleration source with the exponents 2 . The dashed lines show the differential flux formed solely by the accelerated electrons; (b) same as panel (a) for the heating pulse at 18:54 UT.

For both models, $10^{5}$ initial electrons are used for a run of the Monte Carlo code in order to obtain reasonable statistics. Coefficients $A$ in Eqs. (7) and (8) are determined so that the intensity of the $557.7 \mathrm{~nm}$ emission, calculated with the modelling electron flux, is equal to the intensity observed in the experiment.

The electron differential fluxes and the volume emission rates calculated for the first model of the accelerated electrons are presented in Fig. 5a and b for Pulse 1 and Pulse 2, respectively. Left and central panels of these figures present the electron differential flux (the sum of accelerated and thermal electrons) as a function of energy and altitude. The left panel corresponds to an energy of accelerated electrons of $20 \mathrm{eV}$ and the central panel corresponds to an energy of $90 \mathrm{eV}$. The right panels of Fig. 5a and b show the altitude profiles of the enhanced optical emissions calculated with the differential fluxes presented on the left and central panels of the figures. On the plots with the emission altitude profiles, it is clearly seen that they have different dependencies on the energy of acceleration electrons. The profile of $630.0 \mathrm{~nm}$ emission barely changes when the electron energy increases from $20 \mathrm{eV}$ to $90 \mathrm{eV}$. The profiles of other emissions become wider with an increase of the electron energy. Further, for both the $557.7 \mathrm{~nm}$ and $844.6 \mathrm{~nm}$ emissions the magnitudes of their maxima decrease with the energy growth while the magnitude of the $427.8 \mathrm{~nm}$ emission peak decreases.

Figure $6 \mathrm{a}$ and $\mathrm{b}$ presents the modelled electron fluxes versus electron energy. The left panels in these figures show the differential fluxes at the altitude of electron acceleration (230 km for Pulse 1 and $270 \mathrm{~km}$ for Pulse 2). The right panel 

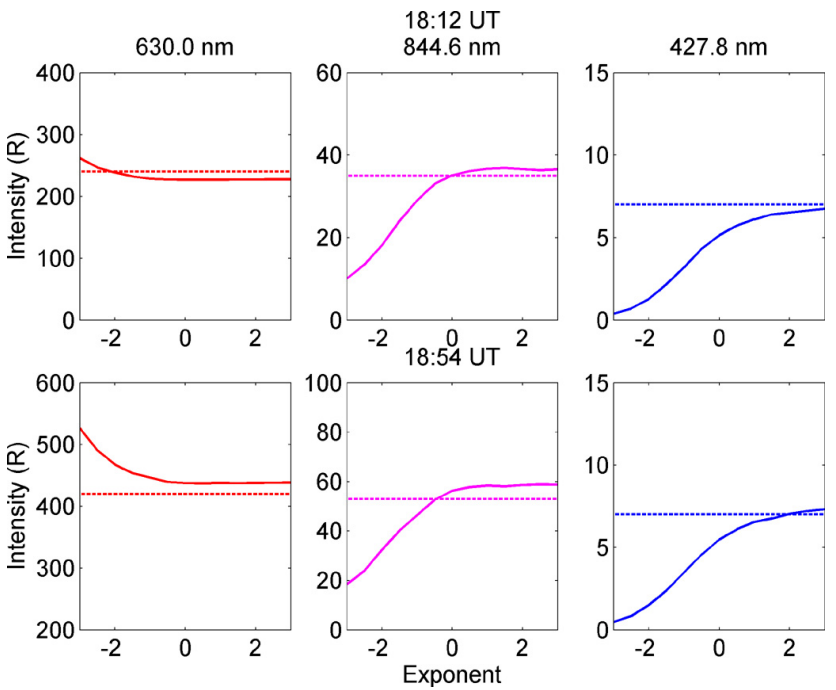

Fig. 10. . Emission intensity dependence on the exponent of the power-law energy spectrum of the electron acceleration source. The top row corresponds to the heating pulse at 18:12 UT. The bottom row corresponds to the heating pulse at 18:54 UT. The solid lines show the calculated emission intensities. The dashed lines indicate the values of the emission intensities observed in the experiment. $I_{6300}^{\bmod } \equiv I_{6300}^{\mathrm{exp}}$.

presents the electron differential fluxes at three different altitudes for an accelerated electron energy of $90 \mathrm{eV}$. The general shape of the electron differential flux is similar to the shape calculated by Gustavsson and Eliasson (2008). The differential flux up to an energy of about $4.5 \mathrm{eV}$ is formed by the thermal electrons. The accelerated electrons are responsible for the remaining part of the spectra while their input to the lower energy electron flux, indicated by dashed lines in the right plots of Fig. 6 is insignificant. But it is striking that the shape of the differential flux at energies less than $E_{0} / 2$ does not depend on the initial energy $E_{0}$ of the accelerated electrons. It implies that in the first instance the electron collisions and transport determine the flux shape, that is to say the electrons do not "remember" their origin.

The emission intensity dependence on the initial energy of the accelerated electrons is shown in Fig. 7. The $557.7 \mathrm{~nm}$ intensity is not plotted because it is constant and is equal to the observed intensity of $43 \mathrm{R}$, in accordance with a normalization of the intensity of the source of the accelerated electrons. Only 427.8 emission with an excitation threshold of $18.75 \mathrm{eV}$ varies noticeably with the initial energy. However, for energies more than $60 \mathrm{eV}$ the intensity of this emission, similar to the others, approaches a constant value. A good agreement, for all emissions, of the levels of the constant intensity with the observed intensities allows us to conclude that the electrons should be accelerated to energies more than $60 \mathrm{eV}$.

The calculation results for the second model of the electron acceleration source are presented in Figs. 8-10. Figure $8 \mathrm{a}$ and $\mathrm{b}$ presents the energy-altitude distribution of dif- ferential electron fluxes and the emission altitude profiles for Pulse 1 and Pulse 2, respectively. The left panels of these figures show the calculation results for the power-law electron acceleration source with the exponent, $\gamma=-2$, and the central panels correspond to $\gamma=2$. Dependencies of the differential electron fluxes on the electron energy for Pulse 1 and Pulse 2 are presented in Fig. 9a and b. The left plots of these figures give the differential fluxes for the different exponents at an altitude of $230 \mathrm{~km}$ for Pulse 1 and at $270 \mathrm{~km}$ for Pulse 2. The right plots show the electron fluxes calculated for the electron acceleration source with exponent $\gamma=2$ at three different altitudes.

Both the shape of the electron flux and the profile of the optical emissions are influenced by the changing shape of the acceleration electron source. However, this dependence becomes insignificant when the exponent is larger than zero. Moreover, the differential electron flux for power-law acceleration source with the exponent larger than zero is almost the same as the flux of the mono-energetic electron source with initial energy more than $60 \mathrm{eV}$. This fact is well manifested in Fig. 10 where the optical emission intensities are plotted versus the exponent. Similar to results for the monoenergetic source of the accelerated electrons, the emission intensity reaches a constant level with increasing exponent. The values of these constant levels are very close to the observed emission intensity. From this fact, we can conclude that for the conditions of our heating experiment, the exponent, $\gamma$, characterising the energy distribution of the accelerated electrons, should be higher than 0.5 . The slight differences between the values of the constant levels for the $630.0 \mathrm{~nm}$ emission for the two sources of the accelerated electrons are explained by uncertainties in the experimental electron density and temperature used for the thermal electron modelling.

\section{Conclusions}

In this study, we applied the Monte Carlo method for the simulation of the transport of lower energy electrons into the upper Earth's atmosphere. The modelling results were used for analysis of the optical emissions enhanced during ionosphere modification by a powerful HF radio wave. For the conditions of the EISCAT heating experiment on 10 March 2002, we obtained the following results:

1. $630.0 \mathrm{~nm}$ emission with an excitation threshold of $1.96 \mathrm{eV}$ is formed predominantly ( $>70 \%$ of $630.0 \mathrm{in}-$ tensity) by thermal electrons, and the accelerated electrons play a minor role in the excitation of this emission;

2. In order to explain the experimentally observed intensities for the emissions with a higher excitation threshold, the electrons must gain an energy of more than $60 \mathrm{eV}$;

3. For the acceleration electron source with the power-law energy dependence $\left(Q(E)=A E^{\gamma}\right)$, the intensities of 
the optical emissions are primarily defined by the total intensity of the accelerated electrons and do not depend significantly on the exponent $\gamma$, if it is greater than 0 .

Acknowledgements. We gratefully acknowledge support for this research from ELSTATIK Foundation, Günter and Sylvia Lüttgens.

Topical Editor K. Kauristie thanks M. Kosch and K. U. Kaila for their help in evaluating this paper.

\section{References}

Adeishvili, T. G., Gurevich, A. V., Lyakhov, S. B., Managadze, G. G., Milikh, G. M., and Shlyuger, I. S.: Ionospheric emission caused by an intense radio wave, Sov. J. Plasma Phys., 4, 721726, 1979.

Bernhardt, P. A., Duncan, L. M., and Tepley, C. A.: Artificial airglow excited by high-power radio waves, Science, 242, 10221027, 1988.

Berrington, K. A. and Burke, P. G.: Effective collision strengths for forbidden transitions in e-N and e-O scattering, Planet. Space Sci., 29, 377-381, 1981.

Biondi, A. A., Sipler, D. P., and Hake, R. D.: Optical (26300) detection of radio frequency heating of electrons in the $\mathrm{F}$ region, $\mathrm{J}$. Geophys. Res., 75, 6421-6424, 1970.

Brändström, B. U. E., Leyser, T. B., Steen, Å., Rietveld, M. T., Gustavsson, B., Aso, T., and Ejiri, M.: Unambigous evidence of HF pump-enhanced airglow, Geophys. Res. Lett., 26, 3561-3564, 1999.

Djuth, F. T., Pedersen, T. R., Gerken, E. A., Bernhardt, P. A., Selcher, C. A., Bristow, W. A., and Kosch, M. J.: Ionospheric modification at twice the electron cyclotron frequency, Phys. Rev. Lett., 94, 125001, doi:10.1103/PhysRevLett.94.125001, 2005.

Gilmore, F. R., Laher, R. R., and Espy, P. J.: Franck-Condon factors, r-centroids, electronic transition moments, and Einstein coefficients for many nitrogen and oxygen band systems, J. Phys. Chem. Ref. Data, 21, 1005-1107, 1992.

Grach, S. M.: On kinetic effects in the ionosphereic F-region modified by powerful radio waves, Radiophys. Quantum El., 42, 572588, 1999.

Gurevich, A. V.: Nonlinear effects in the ionosphere, Usp. Fiz. Nauk, 50, 1091-1121, 2007.

Gustavsson, B. and Eliasson, B.: HF radio wave acceleration of ionospheric electrons: Analysis of HF-induced optical enhancements, J. Geophys. Res., 113, A08319, doi:10.1029/2007JA012913, 2008.

Gustavsson, B., Sergienko, T., Häggström, I., and Honary, F.: Simulation of high energy tail of electron distribution function, Adv. Polar Upper Atmos. Res., 18, 1-9, 2004.

Gustavsson, B., Sergienko, T., Kosch, M. J., Rietveld, M. T., Brändström, B. U. E., Leyser, T. B., Isham, B., Gallop, P., Aso, T., Ejiri, M., Grydeland, T., Steen, Å., LaHoz, C., Kaila, K., Jussila, J., and Holma, H.: The electron energy distribution during HF pumping, a picture painted with all colors, Ann. Geophys., 23, 1747-1754, doi:10.5194/angeo-23-1747-2005, 2005.

Hedin, A. E.: Extension of the MSIS thermospheric model into the middle and lower atmosphere, (The model can be run online at http://ccmc.gsfc.nasa.gov/modelweb/atmos/msise.html), J. Geophys. Res., 96, 1159-1172, 1991.

Holma, H., Kaila, K. U., Kosch, M. J., and Rietveld, M. T.: Recognizing the blue emission in artificial airglow, Adv. Space Res., 38, 2653-2658, 2006.

Itikawa, Y.: Cross sections for electron collisions with oxygen molecules, J. Phys. Chem. Ref. Data, 38, 1-20, 2009.

Itikawa, Y. and Ichimura, A.: Cross Sections for Collisions of Electrons and Photons with Atomic Oxygen, J. Phys. Chem. Ref. Data, 19, 637-651, 1990.

Jackman, C. H., Garvey, R. H., and Green, A. E. S.: Electron impact on atmospheric gases; I. Updated cross-sections, J. Geophys. Res., 92, 5081-5090, 1977.

Kalogerakis, K. S., Slanger, T. G., Kendall, E. A., Pedersen, T. R., Kosch, M. J., Gustavsson, B., and Rietveld, M. T.: Remote Oxygen Sensing by Ionospheric Excitation (ROSIE), Ann. Geophys., 27, 2183-2189, doi:10.5194/angeo-27-2183-2009, 2009.

Kosch, M. J., Pedersen, T., Hughes, J., Marshall, R., Gerken, E., Senior, A., Sentman, D., McCarrick, M., and Djuth, F. T.: Artificial optical emissions at HAARP for pump frequencies near the third and second electron gyro-harmonic, Ann. Geophys., 23, 15851592, doi:10.5194/angeo-23-1585-2005, 2005.

Laher, R. R. and Gilmore, F. R.: Updated excitation and ionization cross sections for electron impact on atomic oxygen, J. Phys. Chem. Ref. Data, 19, 277-305, 1990.

Mantas, G. P. and Carlson, H. C.: Reinterpretation of the $6300-\AA$ airglow enhancements observed in ionosphere heating experiments based on analysis of Platteville, Colorado, data, J. Geophys. Res., 101, 195-200, 1996.

Mishin, E., Carlson, H. C., and Hagfors, T.: On the electron distribution function in the $\mathrm{F}$ region and airglow enhancements during HF modification experiments, Geophys. Res. Lett., 27, 28572860, 2000.

Mishin, E. V., Burke, W. J., and Pedersen, T.: HF-induced airglow at magnetic zenith: theoretical considerations, Ann. Geophys., 23, 47-53, doi:10.5194/angeo-23-47-2005, 2005.

Opal, C. B., Peterson, W. K., and Beaty, E. C.: Measurements of secondary-electron spectra produced by electron impact ionization of a number of simple gases, J. Chem. Phys., 55, 4100-4106, 1971.

Pedersen, T. R. and Carlson, H. C.: First observations of HF heaterproduced airglow at the High frequency Active Auroral Research Program facility: Thermal excitation and spatial structuring, Radio Sci., 36, 1013-1026, 2001.

Porter, H. S., Varosi, F., and Mayr, H. G.: Iterative solution of the multistream electron transport equation, 1 Comparison with laboratory beam injection experiment, J. Geophys. Res., 92, 59335959, 1987.

Ralchenko, Yu., Kramida, A. E., Reader, J., and NIST ASD Team: NIST Atomic Spectra Database (ver. 4.1.0), [Online], available at: http://physics.nist.gov/asd, last access, 11 May 2012, National Institute of Standards and Technology, Gaithersburg, MD, 2011.

Sergienko T. I. and Ivanov, V. E.:. Transport of electrons in atmospheric gases. I - Integral characteristics, Geomagn. Aeron., 31, 635-642, 1991.

Sergienko, T., Gustavsson, B., Steen, Å., Brändström, U., Rietveld, M., Leyser, T., and Honary, F.: Analysis of excitation of the $630.0 \mathrm{~nm}$ airglow during heating experiment in Troms $\varnothing$ on 16 February 1999, Phys. Chem. Earth, 25, 531-535, 2000. 
Sipler, D. P., Enemark, E., and Biondi, M. A.: 6300-Å intensity variations produced by the Arecibo ionospheric modification experiment, J. Geophys. Res., 79, 4276-4280, 1974.

Streit, G. E., Howard, C. J., Schmeltekopf, A. L., Davidson, J. A., and Schiff, H. I.: Temperature dependence of $\mathrm{O}\left({ }^{1} \mathrm{D}\right)$ rate constants for reactions with $\mathrm{O}_{2}, \mathrm{~N}_{2}, \mathrm{CO}_{2}, \mathrm{O}_{3}$, and $\mathrm{H}_{2} \mathrm{O}$, J. Chem. Phys., 4761-4764, 1976.
Swartz, W. E., Nisbet, J. S., and Green, A. E. S.: Analytic expression for the energy-transfer rate from photoelectrons to thermalelectrons, J. Geophys. Res., 76, 8425-8426, 1971.

Tabata, T., Shirai, T., Sataka, M., and Kubo, H.: Analytic crosssections for electron impact collisions with nitrogen molecules, Atomic Data and Nuclear Data Tables, 92, 375-406, 2006. 